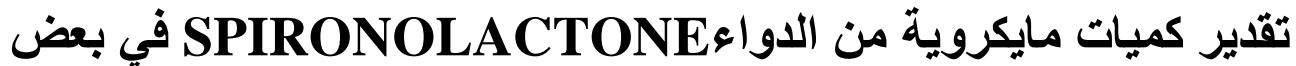 المستحضرات الصيدلانية باستخدام تقنية التألق الجزيئي
}

\author{
فاطمة علي حسبن \\ 2007/9/10 2 ت تاريخ قبول النشر
}

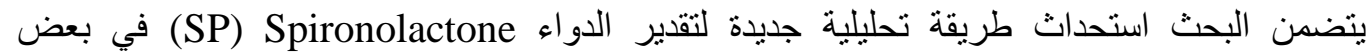

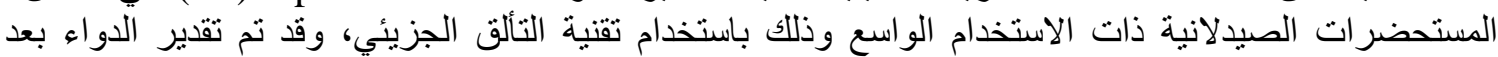
در اسة الظروف العملية الفضلى وأمكن استحصال النتائج الاتية:-

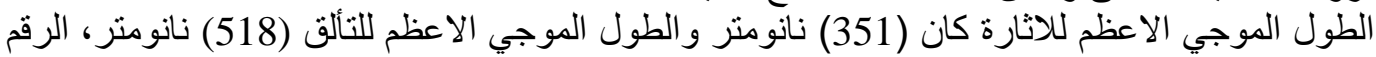

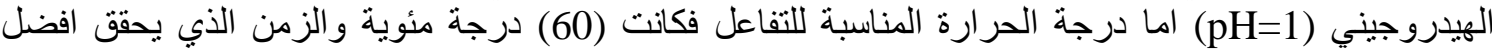

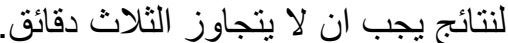
المعطيات التحليلية الاحصائية كانت كما يلي:-

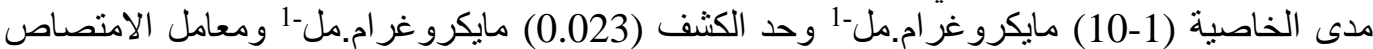

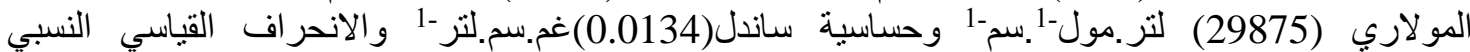

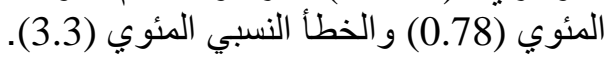

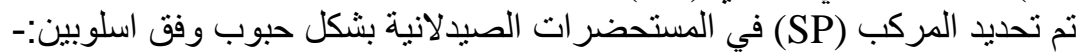

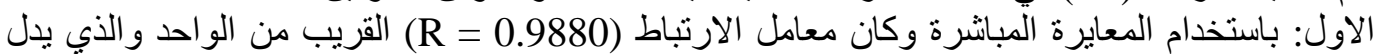

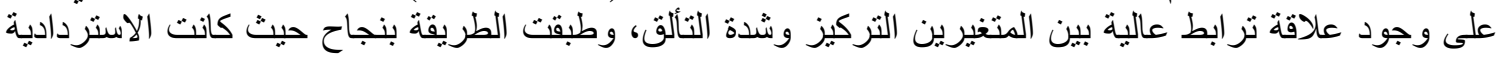
الثاني: باستخدام منحني الاضافات القياسي حيث كان معامل الارتباط (SP) (R = 0.9880) وكانت الاستردادية

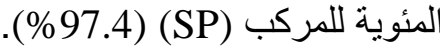
المئوية للمركب (SP) (96.6\%).

المقدمة

Shimadzu Spectrofluorophotometer RFI5OI

2- ميزان رقمي حساس من نوع: - Sart Oriul BL-2105

وكانت الظروف الالية الفضلى للتحليل

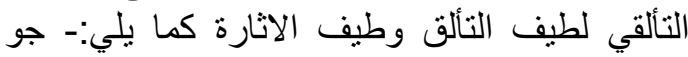

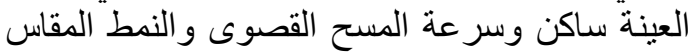

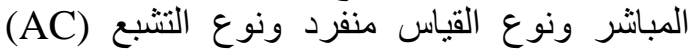
وفي درجة الحر ارة الغرفة.

\section{B - B - المحاليل المستخدمة:} 1- المحلول القياس الام للاواء (SP):

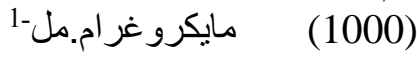

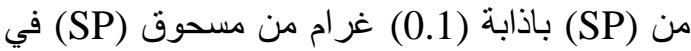
كمية قليل من الايثانول ثم اضيف (4) باذبة من مل من من المحلول (1:1) حامض الكبريتيك بعدها سخن

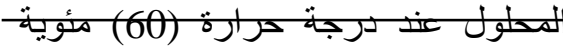

لمدة لاتتجاوز ثلاث دقائق (لوحظ تلون المحلول

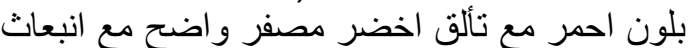
رائحة مميزة لغاز كبريتيد الهيدروجين) بعدها اكمل التعل الحجم الى 100 مل بالايثانول.

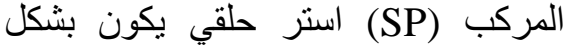
مسحوق ابيض او ابيض مصفر عديم الرائحة غير ذائب في الماء وقليل الذوبان من الايثر ولكنه يذوب الئوب

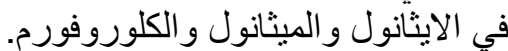
ان الطول الموجي الاعظم للامتصاص الماص

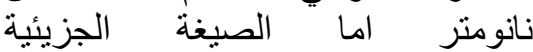
فهي $\left(\mathrm{C}_{24} \mathrm{H}_{32} \mathrm{O}_{4} \mathrm{~S}\right)$ يصنف الدوراء (SP) علاجياً ضمن صنف المدررات وهي الادوية التي تزيد

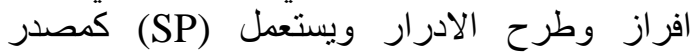
لمعالجة الاستسقاء وقصور القادر القب الاحتقاني وتليف

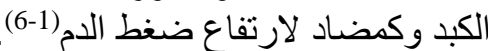

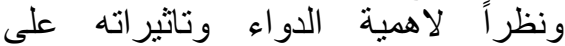

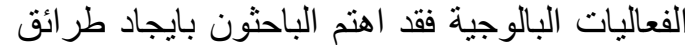

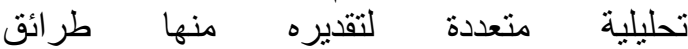
الكروماتو غر افية(7-15) وكذللك بطر ائق طبفية(16-24).

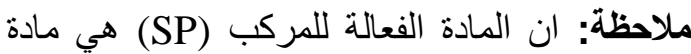
قباسية نقية نم الحصول عليها من معمل هي مادك

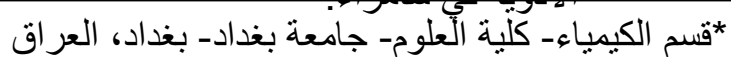

$$
\text { الجزء العملي }
$$
A - الاجهزة المستخدمة: 
بعدها قيست شدة التألق عند الطول الموجي الاعظم

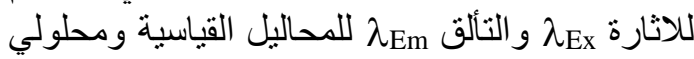

اما بخصوص المستحضر الصيدلاني

فلقد اتبع نفس الاسلوب المبين (ALCTONE) اعلاه في المستحضر الاول.

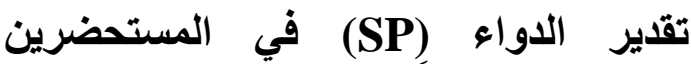
الصيدلانيين تألقياً بطريقة الاضافة القياسية

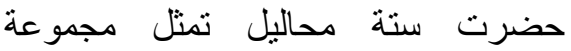

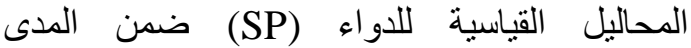

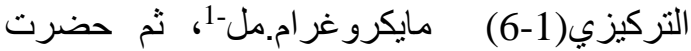

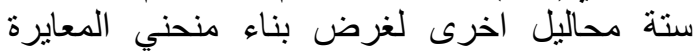
بطريقة اضافات القياس اذ نقلت النقان حجوم تتزاوح )

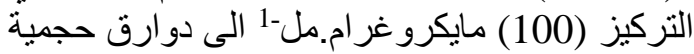

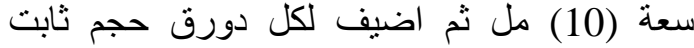
مقداره (0.3) مل من محلول المستحضر الصيدلاني

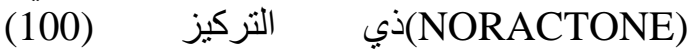

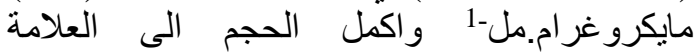
بالايثانول، ضبطت جميع الظروف العملية وقيست الطيت شدة التألق لكل محلول عند الطول الموجي الاعظم للاثارة اما بخصوص المستحضر الصيلاني فلقد اتبع نفس الاسلوب الذي حضر (ALCTONE) به المتحضر الاول.

\section{النتائج والمناقشة}

1- تحديد الطول الموجي الاعظم للاثارة

كان الطول الموجي الاعظم للاثارة الطي والتألق تصدي (351)نانومتر الثنكل (1) في حين كان الطول الطول

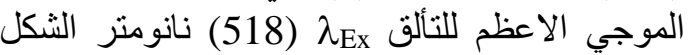

ان الثكل(3) يوضح التفاعلات المؤدية الى الى الثيا

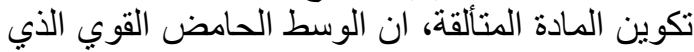

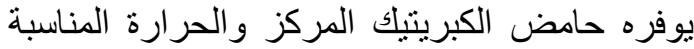

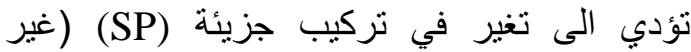

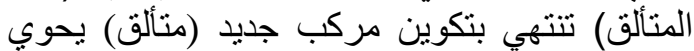
في تركيبه على اواصر مزدوجة منبادلة المسؤولة عن ظاهرة التألق.

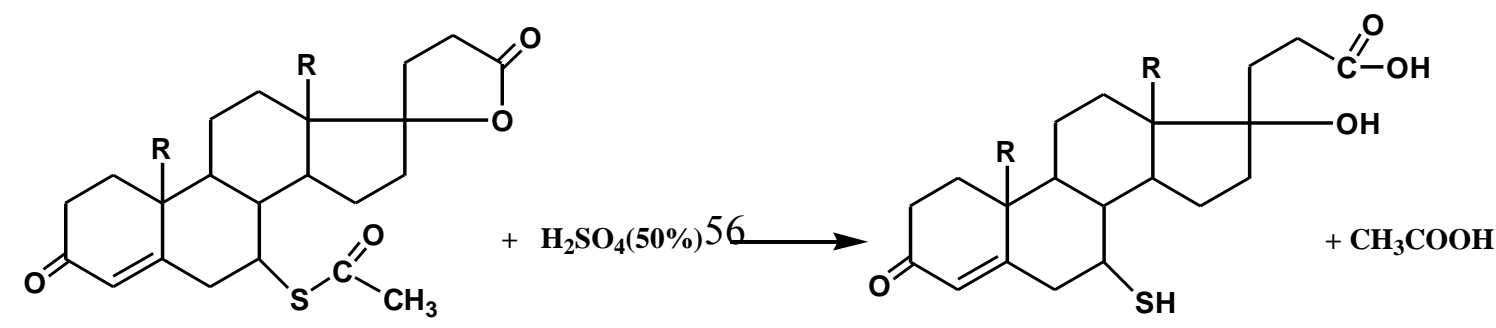

ولغرض الحصول على منحني المعايره

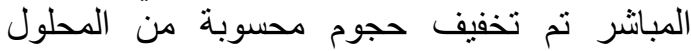
القياس الام بالايثانول وفي دوارق حجمية مناسبة،

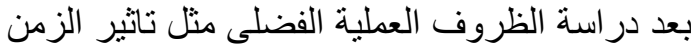
درجة الحرارية وزمن الترشعيع اضافة الى تاثير لتير وجود الماء على طيف التألق.

2- محاليل المستحضر ات الصيلانية: تم استخدام اثثين من الهيلة المستحضرات الصيدلانية لغرض تقدير الدواء (SP) فيهما.

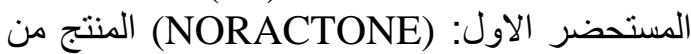
قبل الثركة الاردنية لانتاج الادوية المساهمة

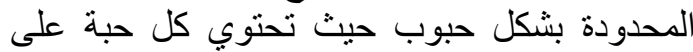

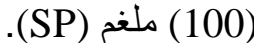

المستحضر الثاني: (ALCTONE) المنتج من قبل كنتجات ميديكو للادويةــ سوريا بشكل حبوب تحتوي كل حبة على (100) ملغم (SP). تم وزن (5) حبات من المستحضر الصيدلاني (NORACTONE) وطحنت في هاون المس حيث تحولت الى مسحوق فكان الوزن الكلي لـ لـ

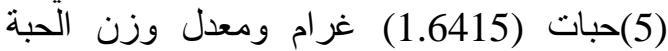
الواحدة (0.3341) غرام فأذا كانت الحبة الواحدة

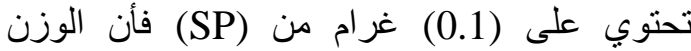
المؤخوذ من المسحوق المكافئ لـ (0.1) غرام من من الرن

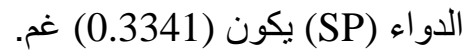
تعامل الكمبة الموزونة اعلاه لغرض

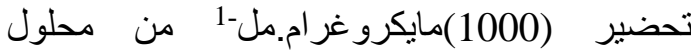
السنتحضر (NORACTONE) كما في الفقرة السابقة عند تحضير المحلول القياسي الام.

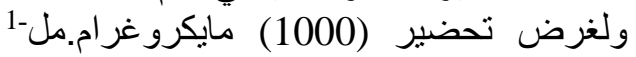

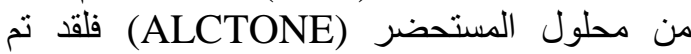
اتباع نفس الاسلوب الذي تم به تحضير المستحضر

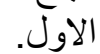

تقدير الدواء (SP) في المستحضرين الصيدلانيين تألقياً بطريقة المعايرة المباشرة حضرت عشرة محاليل تمثل مجموعة المبائل المبرة

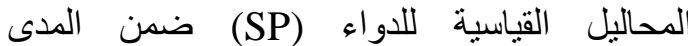
التركيزي (1-10) مايكرو غرام.مل-1-1. نقل الحجمان (0.3 مل و0.6 مل مل من محلول

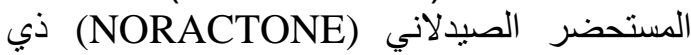

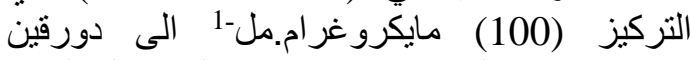

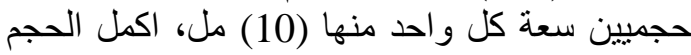
الى العلامة بالايثانول، ضبطت كل الظروف العملية 


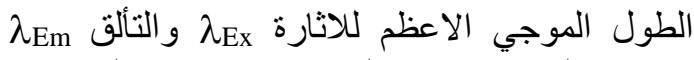
ومدى التركيز ومعامل الامتصاص لاصن المولاري لاري وحساسية ساتدل.

الجدول (1) المعطيات التحليلية لمنحني المعايرة

\begin{tabular}{|c|c|c|c|c|c|}
\hline $\begin{array}{c}\text { Compoun } \\
d\end{array}$ & $\begin{array}{l}\lambda_{\mathrm{EX}} \\
(\mathrm{nm})\end{array}$ & $\begin{array}{c}\lambda_{\mathrm{EM}} \\
(\mathbf{u m})\end{array}$ & $\begin{array}{l}\text { Linearity } \\
\mu \text { g.ml }{ }^{-1}\end{array}$ & $\begin{array}{c}\varepsilon \\
\text { L.mole } \\
{ }^{-1} . \mathrm{cm}^{-1} \\
\end{array}$ & $\begin{array}{c}\text { S } \\
\text { g.cm }\end{array}$ \\
\hline $\begin{array}{l}\text { Sp.Standar } \\
\mathrm{d}^{*}\end{array}$ & 351 & 518 & $1-10$ & 29875 & 0.0139 \\
\hline
\end{tabular}

(SP) Standard* تفاعل SP) Standard في الوسط الحامضي (SP) Standard )

الجدول(2) يبين المعطيات الاحصائية

لمنحني المعايرة المباشرة ويتضمن قيم الخطأ النسبي الاحي

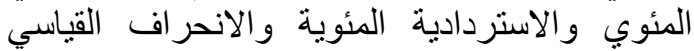

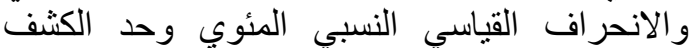
ومعامل الارتباط.

الجدول (2) المعطيات الاحصائية للطريقة التحليلية

\begin{tabular}{|c|c|c|c|c|c|}
\hline \multicolumn{6}{|c|}{ بتطبيق لمنحني المعايرة المباشرة } \\
\hline \%Erel & \% Rec. & S.D & $\%$ RSD & $\begin{array}{c}\text { D.L } \\
\mu \mathrm{g} . \mathrm{ml}^{-1}\end{array}$ & $\mathbf{R}$ \\
\hline 3.3 & 96.6 & 3.6 & 0.78 & 0.023 & 0.9880 \\
\hline
\end{tabular}

نستنتج من قيم هذا الكثف (0.023)

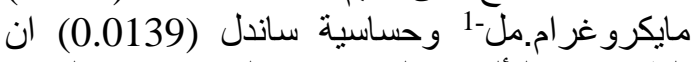

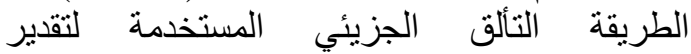
الدواء (SP) ولاول مرة في هذه الدربة استة هي طريقة تحليلية دقيقة وحساسة، وان قيمة معامل الارنباط (RP)

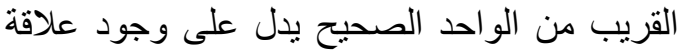
ترابطية عالية بين قيم المتغيرين التركيز وشئ والئ

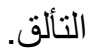

2- انتقاء الظروف العملية الفضلى لتعيين الاواء (SP) بطريقة التحليل التألقي

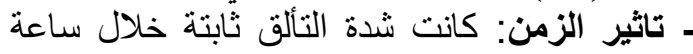

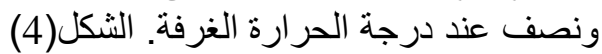

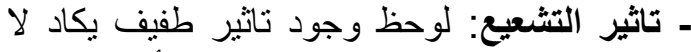
بذكر للاشعة الفوق البنفسجية على شدة تألق المحلول

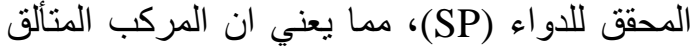
مستقر خلال فترة زمنية قدر ها (30) دقيقة. الثكل

- تاثير درجات الحرارة: ان شدة التألق تنخفض

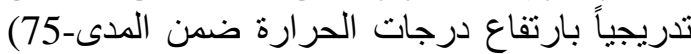

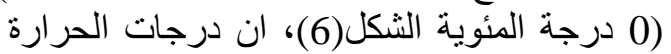

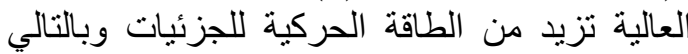

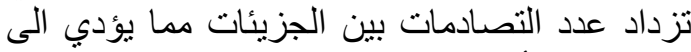

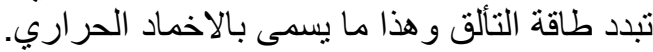

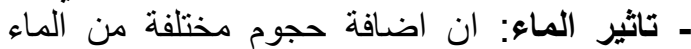

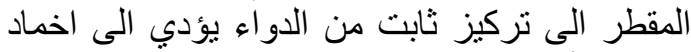

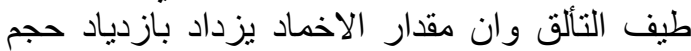
الماء المضاف. الثكل (7).

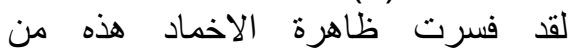
قبل (Donald) (25) و اخرون، بوجود قوى الستقطاب بين المخمد (الماء) و المادة المتألقة او قد يكون فون عزئ

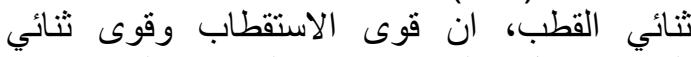

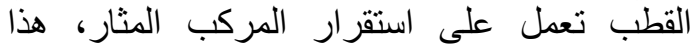
الاستقرار يصاحبه انخفاض من الطاقة.

منحني المعايرة المباشرة لتعيين الدواء (SP) بطريقة التألق الجزئي

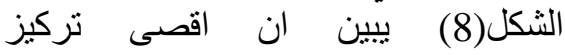

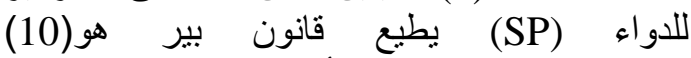
مايكرو غر ام.مل-1 بعدها يبدأ المنحني بالانحر افئ الجدول (1) بيبين المعطيات التحليلية لمنحني المعايرة المباشرة حيث بتضدن الجدول 
وجود تداخلات. يبين الجدول (4) بعض المعطيات التحليلية لمنحني اضافات القياس.

الجدول (4) المعطيات التحليلية لمنحني اضافات القياس

\begin{tabular}{|c|c|c|c|}
\hline $\begin{array}{c}\text { Name of } \\
\text { Pharmaceutical }^{-1}\end{array}$ & $\begin{array}{c}\text { Linearity } \\
\text { mg.ml }^{\mathbf{1}}\end{array}$ & $\begin{array}{c}\text { Start } \\
\text { conc. } \\
\text { mg.ml }^{\mathbf{1}}\end{array}$ & $\begin{array}{c}\text { Found } \\
\text { conc. } \\
\text { mg.ml }^{\mathbf{1}}\end{array}$ \\
\hline NORACTONE & $1-6$ & 3 & 3.1 \\
\hline ALCTONE & $1-6$ & 3 & 3.1 \\
\hline
\end{tabular}

ان الجدول(5) يبين بعض المعطيات الاحصائية لمنحني الاضافات القياسية مثل بعن الخطأ النسبي المئوي والاستئردادية المئوية

والانحراف القياسي النسبي المئوي وحد النئي الكثف ومعامل الارتباط. الجدول (5) المعطيات الاحصائية لمنحني اضافات القياس

\begin{tabular}{|c|c|c|c|c|}
\hline \%Erel & \%Rec & \%RSD & $\begin{array}{c}\text { D.L } \\
\boldsymbol{\mu g . m l}^{-1}\end{array}$ & R \\
\hline 3.3 & 96.67 & 0.78 & 0.024 & 0.988 \\
\hline
\end{tabular}

الاستتناجات

1- يمكن اعتماد الطريقة المستخدمة (طريقة التألق

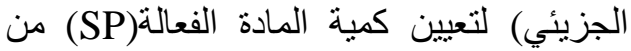
حبوب المستحضر الصبيدة الصياني من منحني المني

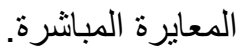
2- تمتاز الطريقة بسر عتها ودقتها و عدم الحاجة الى تصني تحضير ات معقدة و اعطت تكر ارية ودقة عاليتئنين اوضحها النتائج المستحصلة واعطنة

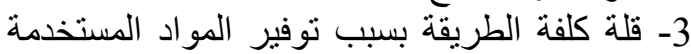
(حامض الكبريتيك و الايثانول و الماء المقطر).

4- لم تحصل تداخلات بين المادة الفعالة المالة

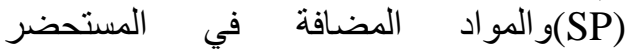
الصيدلاني، هذا ما اوضحه منحني اضافات

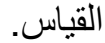

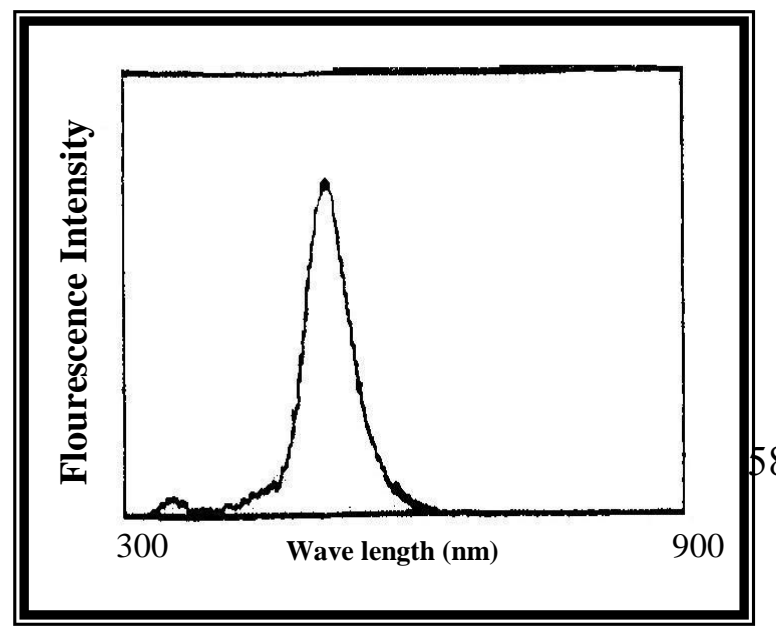

استخدام منحني المعايرة المباشرة قيست شندة التألق لاثنين من المحاليل

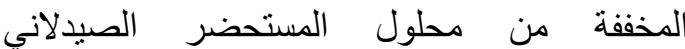
عند الطول الموجي الاعظم (NORACTONE) للاثارة $\lambda_{\text {gm }}$

الجدول(3) الخطأ النسبي المئوي والاستردادية المئوية للاواء (SP) المقدر في المستحضر والاستردية الصيدلاني NORACTONE وفق طريقة للاءئ

\begin{tabular}{|c|c|c|c|c|}
\hline $\begin{array}{c}\text { (SP) } \\
\text { Taker } \\
\mu \text { g.ml }{ }^{-1}\end{array}$ & $\begin{array}{c}\text { (SP) } \\
\text { Found } \\
\mu \mathrm{g} . \mathrm{ml}^{-1}\end{array}$ & $\%$ Erel & $\% \operatorname{Rec}$ & Average \\
\hline $\begin{array}{l}3 \\
6\end{array}$ & $\begin{array}{l}3.1 \\
6.1\end{array}$ & $\begin{array}{c}3.3 \\
1.66\end{array}$ & $\begin{array}{l}96.76 \\
98.34\end{array}$ & $\begin{array}{c}\% \text { Rrel }=2.49 \\
\% \text { Rec }=97.48\end{array}$ \\
\hline
\end{tabular}

ومن النتائج المستحصلة نم حساب النسبة

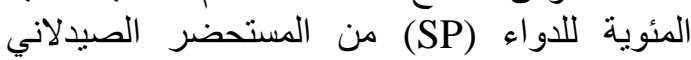
وكانت مطابقة لما هو عليه في دستور الادوية لإنية البريطانية التي تتر اوح بين (98- 105)\% هي

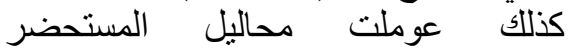
الصيدلاني الاخر (ALCTONE) بنفس الطريقة للمستحضر الاول لغرض تقدير كمبة الدواء (AP)

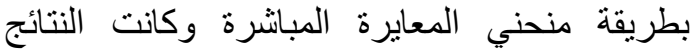
متساوية لكلا المستحضرين الصبدلانيين.

\section{استخدام منحني الاضافات القياسية}

تم رسم منحني الاضافات القياسية وفن العاسية

الثكل(9) و الثكل(10) العائدان للمستحضران

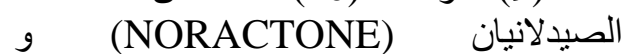
على التو الي. الئن (ALCTONE) ويلاحظ من الثكلين المشار إليهما عدم التئل

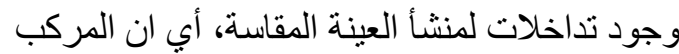
في كلا المستحضرين لايتأثر بالمو اد المضافية المانة

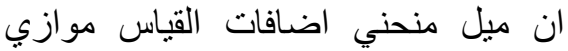

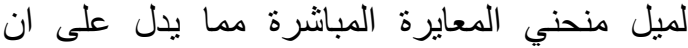
تقدير (SP) بواسطة منحني المعايرة المباثرة كاف الفائ ولاحاجة الى استخدام منحني اضافات القياس لعدم

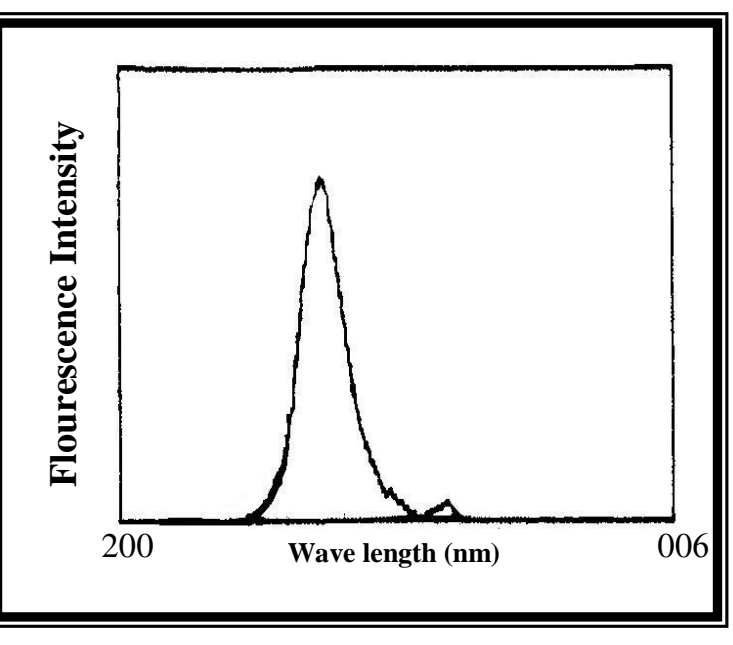



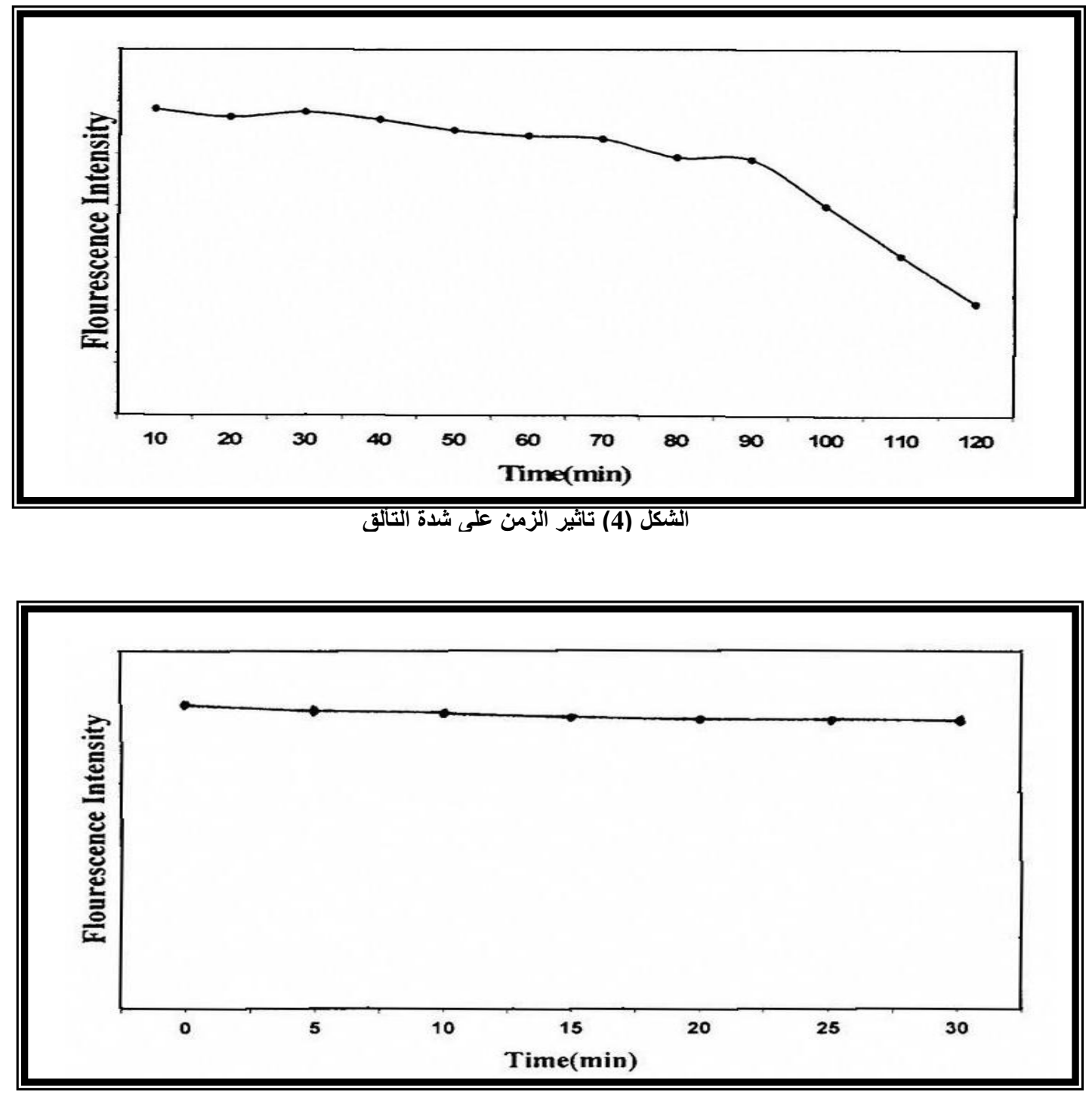

الثكل (5) تاثير التثعيع على شدة التألق خلال 30 دقيقة

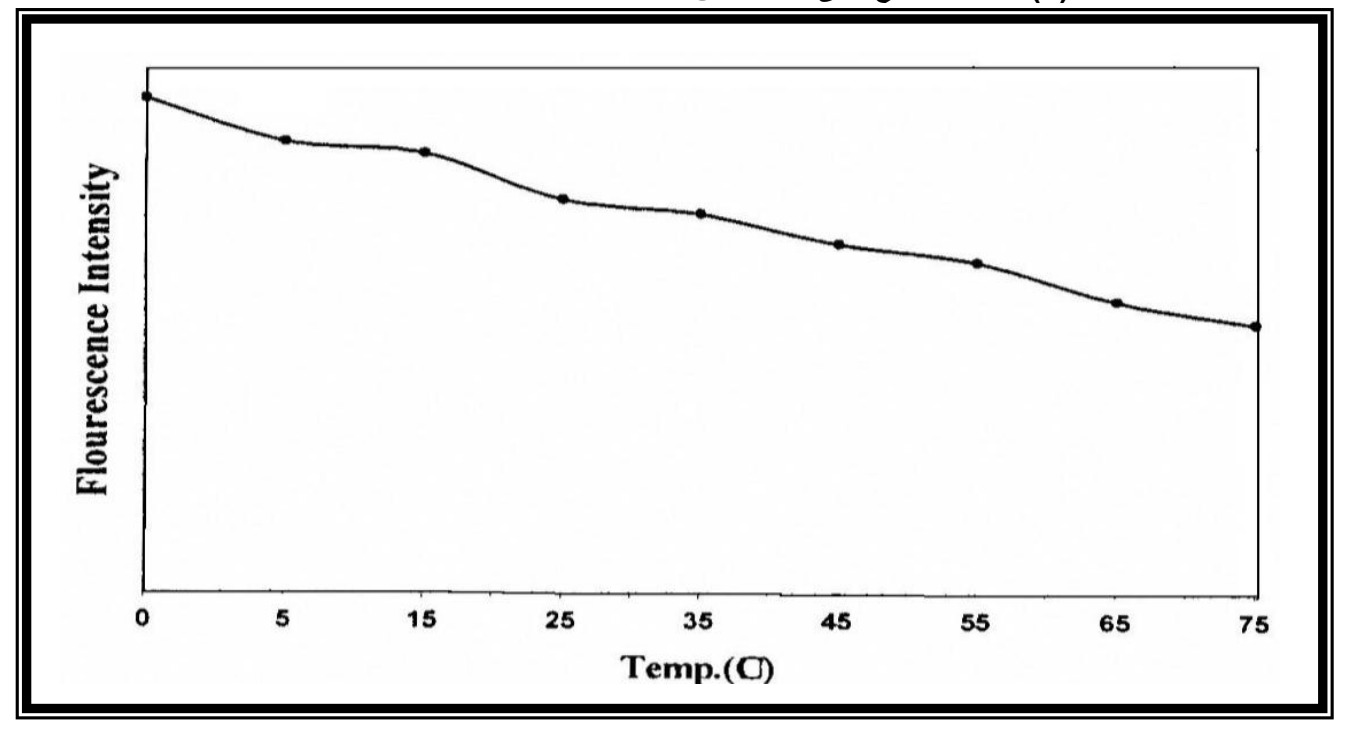




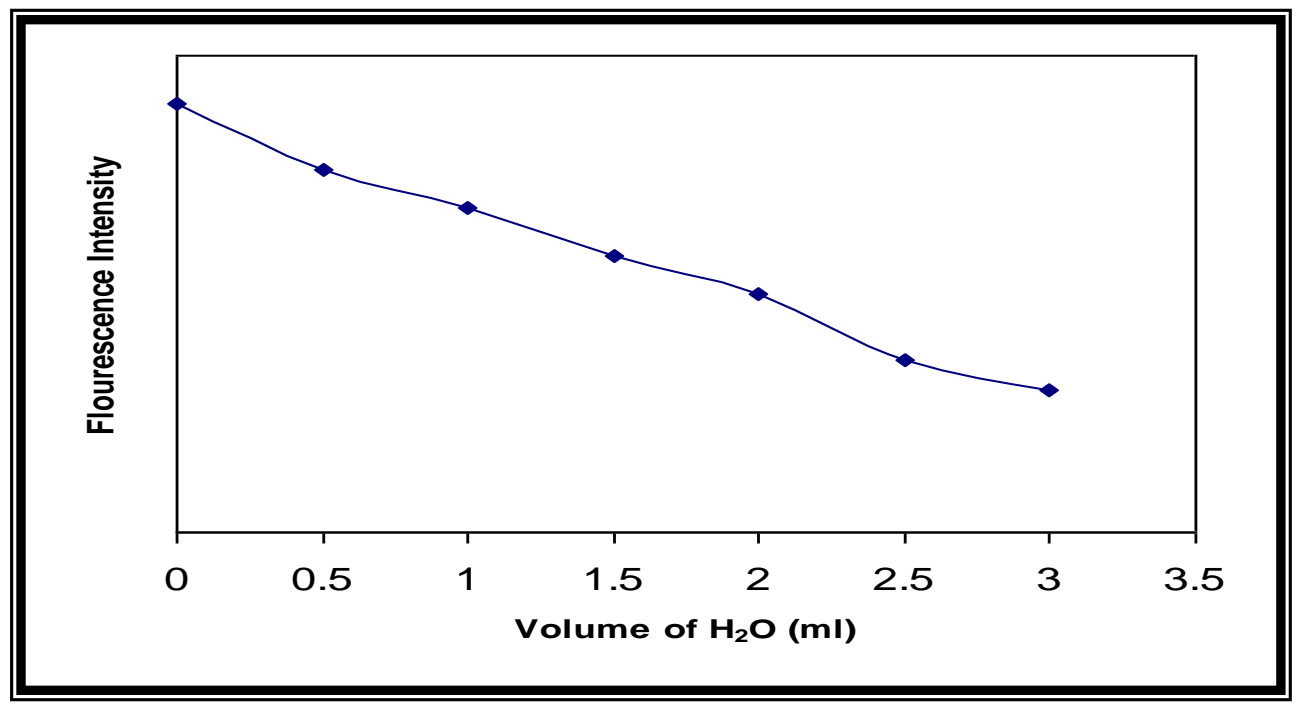

الثكل (7) تاثير الماء على شدة التألق

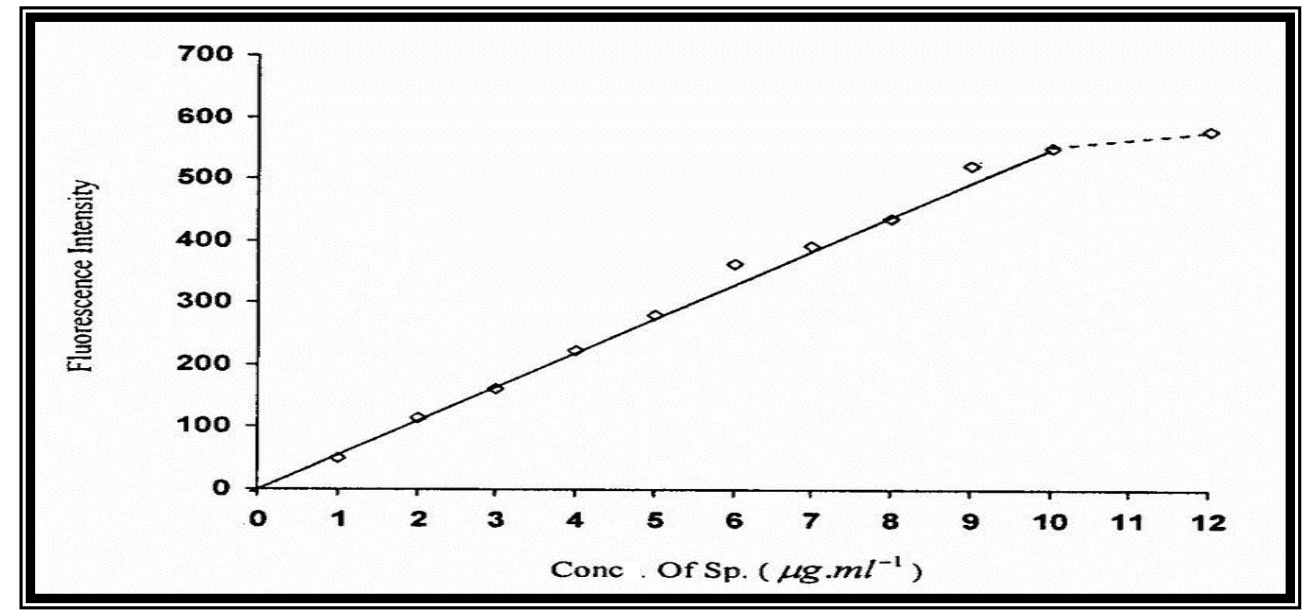

الثكل (8) منحنى المعايرة المباشرة لتعييم الدواء Sp بطريقة التألق الجزيئي

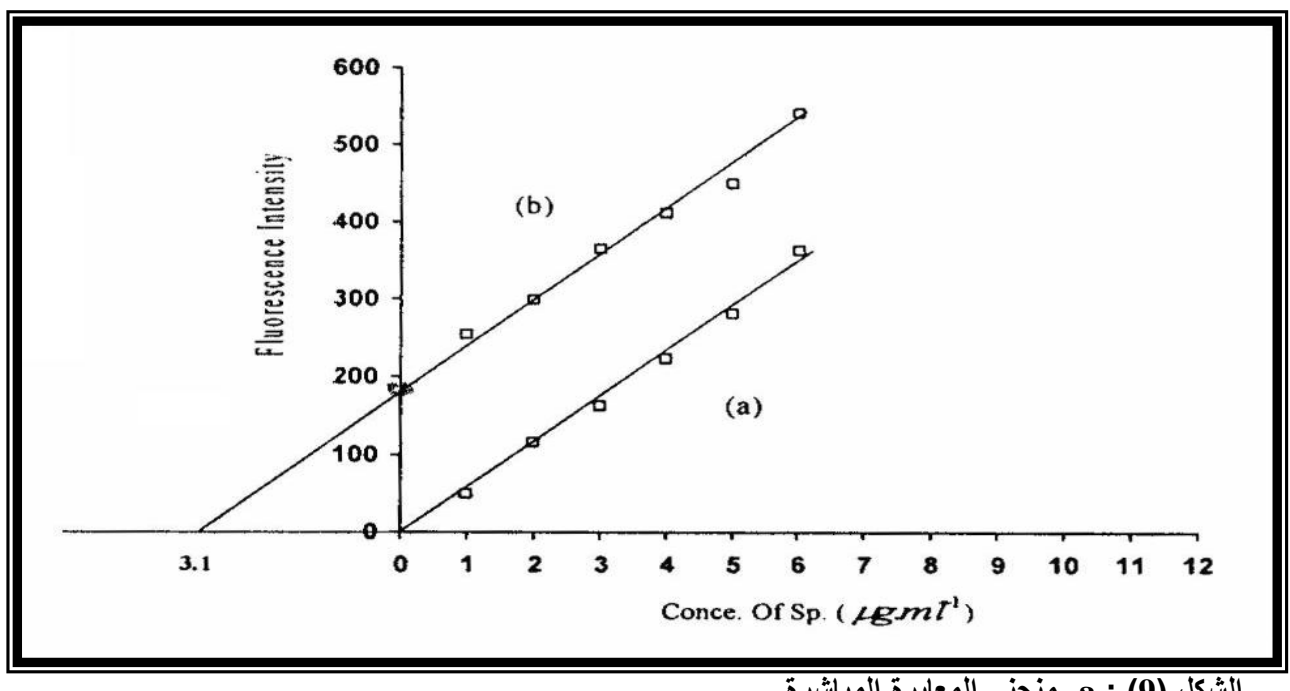

الشكل (9) : a - a منحنى المعايرة المباشرة.

-b منحنى المعايرة بطريقة اضافة القياس لتقلير الدواء Sp في المستحضر الصيلاني 


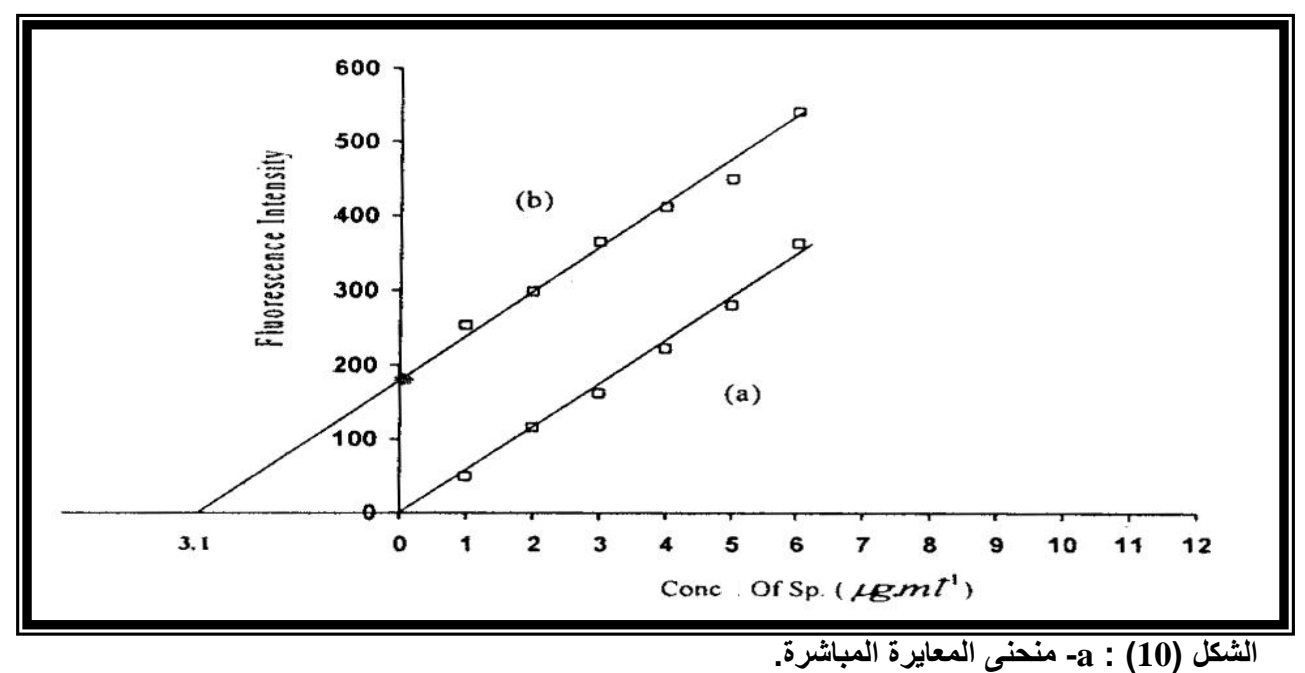

b- منحنى المعايرة بطريقة اضافة القياس لتقدير الدواء Sp في المستحضر الصيدلاني ALCTONE.

10. Besendelder, E. and Endete, R.J., (1981), J., High. Resolut. Chromatogr. Commun, 4(8): 419-421.

11. Decroo, F., Vander Bossche, W., De Moerloose, P., (26 Jul.1985), J. Chromatogr., 329(3): 422-427.

12. Shrry, J. H., O'Dannell, J. P. and Colby, H.D., (10 Jun.1986), J. Chromatogr., Biomed Appl., 47(1): 183-190.

13. Prased, T.N.V., Roa, E.V., Sastry, C.S.P. and Roa, G.R., ( Apr 1987), Indian drugs, 24(7): 346-350.

14. Bonet-Domingo-E., MedinaHernandez-MJ, Garcia AlvarezCoque-MC., (1993), J. Pharm. Biomed Anal., 711-716.

15. Alexsander-KS., Vangala-SS., Dollimore-D., (1998), Drug Development and Industrial Pharmacy, 24(2): 101-107.

16. Wessinger, W. and Anterhoff, H.A., (1979), 119(35): 1377-1380.

17. Lodge, B.A., and Lanonetle, M.C., (1979), J.Pharm. Sci., 14(2): 53.

18. Shingbal, D.M., and Barad, U.G., (1984), Indian drugs, 22(3): 163.

\section{Reference}

1.Didebrg; Dupont -Act crystallogr. Sect., (1972), B28, 3014.

2. Martindal, edited by Kathleen Parfitl, (1999), $32^{\text {nd }} E d$.

3.Mary, J. M., Rionard, A.H., and Pameia, C. C., (2000), Pharmacology, $2^{\text {nd }}$ Ed., Lippinocott Williams and Willicins. Pniladelphia, PA @.

4. "The Merk Index on CD-ROM", (2000), $12^{\text {th }}$ Ed. Copyright by Merk Co. Inc., Whitehouse Station, NJ, USA.

5. British Pharmacopia on CD-ROM, (2000), Vol.1.

6.John, M., Savisonn's Principles and practice of medicine, (1981), $3^{\text {rd }}$ Ed., Churchill living stone.

7.Das Gupta, V., and Ghaneker, A.G., (1978), J. Pharm., Sci., 67(6), 889-891.

8. Boreham, D.R, Vose, C.W., Brooks, C. J. W., and Balasubramaninan, V. J., (1978), J., Chromatogr., 153(1):63-75.

9. Neurath, G.B. and Ambrosins, D.J., (1979), J. Chromatogr., 163(2): 
23. Parimo. P., Bharathi.A., and Padima.K., (33 Apr. 1996), Indian drugs, 160-162.

24. Fadi. J. J., (2002), Higher Diploma in Pharm. and Chem. Anal., Thesis, Baghdad University.

25. Donald R.J. and Selinger B.K., (1972), Aust. J. Chem., 25: 897.
19. Moussa, B.A., E1 Kousy, N., (1985), M-Pharm. Weekbl, Sci. Ed, 7(2): $79-82$.

20. Kamal Purlcar, O.S., and Merezes, C., (1985), Indian drugs, 22(7):389 - 390.

21. Shinghal, D.M., and Roa.V. R., (1986), Indian drugs, 23(4), 232-233.

22. Shinghal, D.M., (1987), Indian drugs, 24(9): 450-451.

\title{
Determination Of Micro Amount Of Spironolactone In Some Of Pharmaceutical Preparate By Using a Molecular Luminescence Technique.
}

\author{
Fatima Ali Hossain* \\ *Department of Chemistry, College of Science, University of Baghdad, Jadriya, \\ Baghdad, Iraq.
}

\begin{abstract}
The present study include a new developed method of analysis for determination of drug Spironolaction (SP) in some Pharmaceuticals by Spectrofluorometric method.

Spironolaction was determined under optimal experimental condition that follows :-

The excitation spectrum was $(\lambda=351 \mathrm{~nm})$, the emmetion spectrum was $(\lambda=518$ $\mathrm{nm}), \mathrm{pH}=1$, the suitable temperature for reaction $60^{\circ} \mathrm{C}$ and the optimal time less than (3) minute. The analysis and rang statistical data was:-Linear dynamic rang (1-10) $\mu \mathrm{g}^{\mathrm{m}} \mathrm{ml}^{-1}$, the detection limit (D.L $\left.=0.023 \mu \mathrm{g} \cdot \mathrm{ml}^{-1}\right)$, Molar absorptivity $\left(\varepsilon=29875\right.$ liter mole $^{-1}$ $\left.\mathrm{cm}^{-1}\right)$, Relative standard deviation $(\% \mathrm{RSD}=0.78),(\%$ Erel $=3.3)$ and recovery $(\mathrm{Rec}=$ 96.6) percentage.

Determination of Spironolactone was accomplished by two methods

(A) Using direct calibration curve method, Corr. Coef. $(\mathrm{R}=0.988$ and $\% \mathrm{Rec}=97.4)$.

(B) Using standard additional methods Corr. Coef $(\mathrm{R}=0.988$ and \% Rec $=96.6)$.
\end{abstract}

